\title{
LUT
}

University

\section{Acoustic Detection of Cracks and Delamination in Multilayer Ceramic Capacitors}

Levikari Saku, Kärkkäinen Tommi J., Andersson Caroline, Tamminen Juha, Silventoinen Pertti

This is a Author's accepted manuscript (AAM) version of a publication published by IEEE

in IEEE Transactions on Industry Applications

DOI: $\quad 10.1109 /$ TIA.2018.2873989

Copyright of the original publication: (C) 2018 IEEE

Please cite the publication as follows:

Levikari, S., Kärkkäinen, T. J., Andersson, C., Tamminen, J., Silventoinen, P. (2018). Acoustic Detection of Cracks and Delamination in Multilayer Ceramic Capacitors. IEEE Transactions on Industry Applications. DOI: 10.1109/TIA.2018.2873989 


\title{
Acoustic Detection of Cracks and Delamination in Multilayer Ceramic Capacitors
}

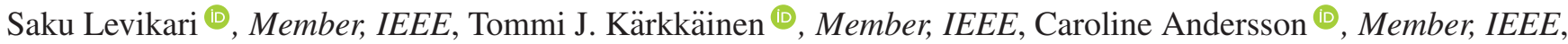 \\ Juha Tammminen, and Pertti Silventoinen, Member, IEEE
}

Abstract-Multilayer ceramic capacitors (MLCC) are the most widely used capacitor type in the electronics industry. However, the brittle ceramic dielectric makes MLCCs prone to mechanical damage. Manufacturing defects or damage during board assembly may cause a capacitor to prematurely fail during its operational life. Here, we demonstrate the fast and non-destructive acoustic screening of MLCCs. Soldered 2220-sized MLCCs were subjected to ac voltage frequency sweeps, causing them to vibrate mechanically. Acoustic responses of the capacitors were measured before and after subjecting the test circuit board to severe bending. The results show that the cracks and delaminations caused by bending induce characteristic changes in the capacitors' acoustic response. A support vector machine classifier was trained to successfully detect damaged capacitors based on their acoustic response.

Index Terms-Acoustic emission, ceramic capacitors, nondestructive testing.

$\mathbf{M}$ ULTILAYER ceramic capacitors (MLCCs) are commonly used in the electronics industry [1]. The ceramic dielectric gives MLCCs high capacitance per volume, but also makes them prone to mechanical damage.

Voids and delaminations are typical manufacturing defects in MLCCs, often related to thermal stresses [2], [3]. Mechanical stress, such as improper printed circuit board (PCB) handling during assembly, can lead to cracks or delamination in MLCCs [4], [5]. Mechanical damage in MLCCs is often left unrecognized during production or assembly, as the capacitor may operate normally electrically. However, in the field, a crack or delamination in an MLCC may grow in size, resulting in loss of

Manuscript received April 5, 2018; revised August 17, 2018; accepted August 29, 2018. This work was supported in part by the TEKES under project Finnish Solar Revolution and in part by Academy of Finland under Grant 278496. Paper 2018-PEDCC-0324.R1, presented at the 2017 IEEE International Symposium on Diagnostics for Electrical Machines, Power Electronics and Drives, Tinos, Greece, Aug. 29-Sep. 1, and approved for publication in the IEEE TRANSACTIONS ON INDUSTRY APPLICATIONS by the Power Electronic Devices and Components Committee of the IEEE Industry Applications Society. (Corresponding author: Saku Levikari.)

S. Levikari, T. J. Kärkkäinen, and P. Silventoinen are with the LUT School of Energy Systems, Lappeenranta University of Technology, 53850 Lappeenranta, Finland (e-mail: saku.levikari@lut.fi; tommi.karkkainen@lut.fi; pertti.silventoinen@lut.fi).

C. Andersson is with ABB Corporate Research Center, 5405 Baden-Dättwil, Switzerland (e-mail: caroline.andersson@ch.abb.com).

J. Tamminen is with ABB Drives, FI-00381 Helsinki, Finland (e-mail: juha. tamminen@fi.abb.com).

Color versions of one or more of the figures in this paper are available online at http://ieeexplore.ieee.org.

Digital Object Identifier 10.1109/TIA.2018.2873989 capacitance, shortened lifespan, or an open or short contact [6]. Larger case size capacitors, used in, e.g., power electronics, have shown to be more prone to damage from PCB bending than the more commonly used cases in the 0402 to 1206 range [7]. Testing the capacitors at the assembly line of the system would prevent unexpected damage in the field. At this moment, however, a suitable quality assurance, or production testing method does not exist.

Defects or damage in MLCCs can be detected by mechanical microsectioning [8] and chemical etching [9], but these methods are destructive for the capacitor and are therefore unsuitable for production testing. Recently, an X-ray imaging method with sufficient accuracy for reliable crack detection has been proposed [10]. Delamination defects, however, cannot be seen in $\mathrm{X}$-ray images. Other proposed nondestructive methods include ultrasound imaging [5], acoustic emission counting using a mechanical ram [6], impedance analysis under dc bias [11], leakage current monitoring [12], laser speckle pattern analysis [13], opto-acoustic microscopy [14], and neutron radiography [15].

In this paper, the quality assurance testing goal is approached by measuring the acoustic phenomena of the MLCCs. When under ac voltage, MLCCs generate acoustic emissions. Acoustic emissions are physical vibrations caused by the piezoelectric properties of the dielectric $\left(\mathrm{BaTiO}_{3}\right)$ [16], [17]. Recently, it was shown that a narrow-pulse frequency sweep signal can be used to produce vibration in MLCCs, and that bending the circuit board in order to produce cracks in the MLCCs changes the acoustic emission characteristics of the capacitors [18]. Similar findings have been made by Johnson et al. using resonant ultrasound spectroscopy [19]-[21]. The acoustic approach is particularly interesting, since there is a need in the industry for a method that could identify damaged capacitors from an assembled board. Acoustic monitoring has also been applied for other electronic components, such as power semiconductor modules [22]-[24].

The term acoustic emission does not necessarily refer to audible sounds in the human hearing range. Indeed in this context, the authors use the term as a synonym for vibration occurring in the capacitor, caused by the electromechanical phenomena within the capacitor itself.

In this study, acoustic signals produced using the method from [18] are measured using a point contact sensor. Acoustic measurements are conducted before and after bending the circuit board. The measured waveforms are then analyzed and correlated against bending-induced damage in the capacitors. In order to determine the suitability of acoustic measurements for 
TABLE I

EXCITATION SWEEP SIGNAL FOR MLCCS

\begin{tabular}{lr}
\hline Type & Pulse wave \\
Duration & $100 \mathrm{~ms}$ \\
Frequency range & $100 \mathrm{~Hz} \ldots 2 \mathrm{MHz}$ \\
Voltage & $-10 \mathrm{~V} \ldots+10 \mathrm{~V}$ \\
Duty cycle & $80 \%$ \\
Frequency slope & linear \\
\hline
\end{tabular}

detection of physical damage, a support vector machine (SVM) [25] is trained on the acoustic data to classify the MLCCs as either damaged or undamaged. The key observation from the data is that the changes in the acoustic emission correlate quite well to delamination of the end termination in the capacitors, better than to the existence of cracks. This suggests that it may be possible to develop a delamination detection method based on acoustic emission. Such a method would be very welcome, because at the moment a non-destructive test method for finding delamination in MLCCs does not exist. The acoustic measurement may be useful in identifying cracked capacitors as well, even though the correlation in the analyzed signals is not as good as it is for delamination.

This paper has been improved from its original form, published in the SDEMPED 2017 conference [26].

\section{EXPERIMENT}

The experiment was conducted on 602220 -sized MLCC capacitors of $22 \mu \mathrm{F}$, assembled on a purpose-built PCB. Half of the capacitors were flexible end termination capacitors, while the others had "normal" end terminations.

The experimental procedure was as follows: first, the capacitors were characterized individually by driving them with frequency-swept voltage while measuring their acoustic emissions using a point contact sensor. The test board was then subjected to a controlled bending to damage the MLCCs. After bending, the capacitors were re-characterized acoustically. Xray imaging and cross-sectioning were used to obtain accurate information on the actual condition of each capacitor.

After obtaining all the data described above, changes observed in the acoustic data were compared to the damage observed in each capacitor. In addition to 2220-sized MLCCs, the effect of circuit board bending has also been acoustically observed in case of sizes 1206, 1210, and 1812, all of which yield stronger acoustic response than 2220 [27]. Because PCB bending-caused flex cracks have been an issue especially with larger case sizes, this study was performed on 2220-size capacitors. The acoustic data, alongside X-ray and cross-sectioning results, are publicly available [28].

\section{A. Acoustic Characterization}

To obtain an acoustic response, the MLCCs were driven with 9 a pulse frequency sweep signal specified in Table I. A KRN Services KRNBB-PC broadband point contact sensor was attached to the capacitor being characterized (see Fig. 1). The signal was amplified using a KRN AMP-1BB-J measurement amplifier and then recorded using a Keysight MSO-X 4104A

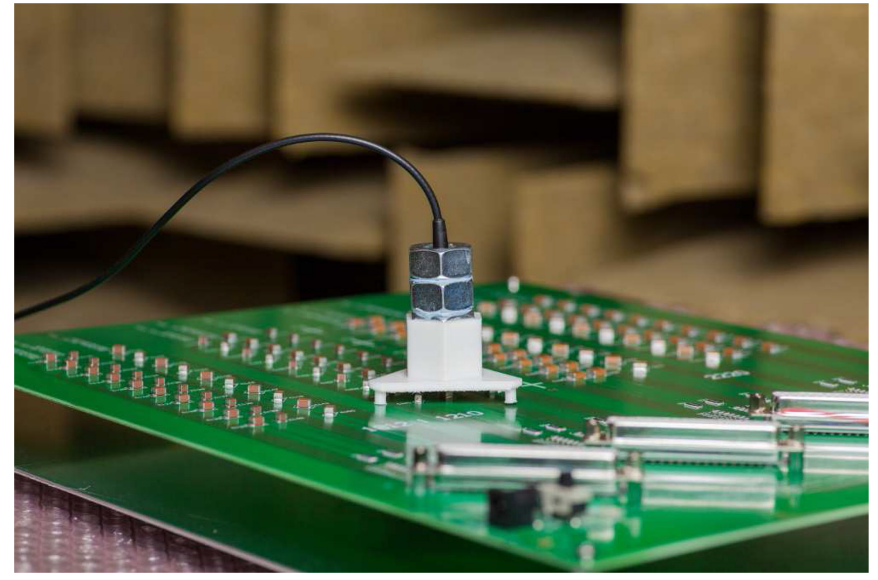

Fig. 1. Point contact sensor placed on top of a capacitor. The sensor is within a 3-D printed fixture on the test $\mathrm{PCB}$, allowing the sensor to be in place without holding it manually.

oscilloscope. The test was conducted in an anechoic room in 124 order to minimize any external acoustic disturbances. 125

The signal from the point contact sensor contains a significant 126 amount of electromagnetic interference (EMI). The harmonics 127 of the pulsed input signal also excite the resonant frequencies of 128 an MLCC to appear several times during a sweep [18]. More- 129 over, at frequencies below $50 \mathrm{kHz}$, a high-amplitude burst likely 130 related to PCB vibration occurred in most of the measurements. 131

To remove the harmonics and EMI, the signals were processed 132 using wavelet decomposition and low-pass filtering. After these 133 stages, the envelope curve of the signal was calculated. The en- 134 velope curve is treated as the acoustic response of the capacitor 135 because it is more convenient to compare envelopes than raw 136 acoustic responses. 137

The acoustic characterization process was conducted in an 138 identical manner before and after bending.

\section{B. Inflicting Damage to the Capacitors}

The test board was bent with a Zwick-Roell Z010 four-point 141 bending setup. Under 18-mm bending displacement, the PCB 142 was subjected to strain levels between 5800 and $8000 \mu \mathrm{Str} 143$ measured at the board centerline, with an average of $6000 \mu \mathrm{Str} . \quad 144$

In reality, a PCB does not experience uniform bending in one 145 direction, especially directly aligned with the orientation of the 146 capacitor. To take this into account, capacitors were assembled 147 in three different angles relative to the direction of the bending. 148 This affects the type and location of damage within the capacitor. 149

\section{X-Ray Imaging and Cross-Sectioning}

After the test board bending, the capacitors were inspected 151 for cracks by X-ray imaging using a Phoenix Nanomex ma- 152 chine. The capacitors were also cross-sectioned to reveal any 153 delamination, which cannot be seen by X-ray. This was done 154 by casting the MLCCs in two-phase epoxy, grinding them with 155 a Struers Rotopol-11 to a desired depth, and then polishing the 156 surface for inspection with a Leica M205C optical microscope. 157 
Cross-sectioning gives accurate information of the condition in the depth that the capacitor has been ground to. If a crack or delamination can be seen in this depth, the capacitor is classified as being damaged. If no damage is seen, however, the existence of damage cannot be ruled out, since a crack or delamination can reside deeper in the capacitor. For this reason, capacitors that showed no damage but exhibited slight changes in their acoustic characteristics were imaged at three different depths to get better understanding of the condition.

The authors would like to point out that cross-sectioning is a very labor-intensive process. It is not feasible to do a complete cross-sectioning of every capacitor at multiple depths. For this reason, full-depth cross-sectioning was not performed for every capacitor.

\section{SVM Classification}

Intuitive discrimination between damaged and undamaged capacitors by inspecting the acoustic responses is difficult and infeasible. Instead, the classification must be based directly on features of the acoustic data. Because each capacitor will yield different numeric features, reliable classification based on strict conditional expressions would be impracticable. Therefore, the machine learning approach is more suitable for the classification task. SVM are machine learning based binary classifiers that have been successfully utilized in signal classification tasks [25], [29], [30]. In the basic SVM approach, the data consist of samples (in this case, the MLCCs) belonging in either positive (1) or negative (0) category. From each sample, $n$ numerical features are extracted. The samples are then mapped onto an $n$ dimensional feature space, where each dimension corresponds to a certain feature. The SVM classifier is then trained by fitting a decision plane into the sample points such that it separates the points in the positive and negative categories by the largest possible margin. Often in real-world scenarios, the data are not linearly separable, i.e., points in different categories overlap. In such a situation, the data points may be mapped into a transformed feature space using a nonlinear kernel function. Furthermore, a cost function is often employed: data points on the wrong side of the decision surface correspond to a total cost, which is minimized as a part of the decision plane fitting [25].

For the task of classifying capacitors, the SVM was trained by extracting only two features from the raw data. This approach kept the classifier model simple and helped in preventing overfitting. Additionally, two-dimensional feature data were convenient to visualize and use in conjunction with the statistical analysis and visual inspection of acoustic responses.

\section{RESULTS}

All the acoustic emission envelopes contained a strong resonance peak at circa $500 \mathrm{kHz}$, related to the fundamental resonance modes of the capacitor body. The authors refer to this peak as the main peak of the capacitor. As can be seen in Fig. 2, the value of this peak is almost doubled between the measurements for a capacitor with no damage. This change seems very significant at first, but further results suggest that the doubled value is still not very large. The increase in the peak value may be explained by better sensor-to-capacitor contact during 212 the second measurement. Another probable explanation is that 213 the $6000 \mu$ Str bending actually caused some degree of physical 214 changes that were missed during the X-ray inspection and mi- 215 crosectioning, since cracks and delaminations can be difficult 216 to detect from the images. Some changes can be seen at fre- 217 quencies lower than that of the main peak, but they are not as 218 significant.

In a capacitor which showed significant delamination in the 220 cross-sectioning (see Fig. 3), the changes are much more pro- 221 nounced. The main peak value is increased to over four times that 222 of a typical value for a pre-bending measurement. Even though 223 not all delaminated capacitors exhibited values this high, no- 224 tably increased main peak values were observed in the majority 225 of them. On the other hand, most of the capacitors did not show 226 delamination as significant as in Fig. 3(b), where a clear gap can 227 be seen extending horizontally across the end termination. 228

At lower frequencies, the changes are comparable to those 229 of an undamaged capacitor. New resonance peaks appear at 230 frequencies higher than that of the main peak, but compared to 231 the main peak, their value is very low. 232

The main peak of a capacitor with cracks (see Fig. 4) exhibits 233 changes even smaller than the intact capacitor. The cracked 234 capacitor does, however, exhibit a new resonance peak at ca. 235 $800 \mathrm{kHz}$. The emergence of such a peak was observed in multi- 236 ple cracked capacitors. The frequency of this peak varies from 237 capacitor to capacitor, and is likely to be affected by the size 238 and location of the crack. Nevertheless, the overall emergence 239 of this peak, referred to as the secondary peak, was observed 240 to correlate with the presence of damage. The crack shown in 241 Fig. 4(b) is approximately $500 \mu \mathrm{m}$ in length and $5 \mathrm{~mm}$ in width, 242 extending across the capacitor end termination. Since this crack 243 causes noticeable changes in the capacitors acoustic response 244 [see Fig. 4(a)], it is very likely that also smaller cracks and 245 delaminations cause acoustically observable changes. 246

To study these peaks and the related damage statistically, the 247 main and secondary peak values were obtained programmati- 248 cally for every acoustic measurement. For this purpose, the main 249 peak value was defined as the highest peak value at frequencies 250 lower than $700 \mathrm{kHz}$. The secondary peak value, respectively, 251 was defined as the highest peak value at frequencies higher than 252 $700 \mathrm{kHz}$. The cross section and X-ray images of every capacitor 253 were examined, and each capacitor was deemed to either have 254 or not have a crack, and to either have or not have delamination. 255

These results were then used to determine which peak indi- 256 cates which type of damage (see Fig. 5). It is evident that the 257 higher the main peak value, the more likely a capacitor is to have 258 delamination damage. The secondary peak value, on the other 259 hand, does not exhibit such a direct relation to the emergence of 260 delamination. 261

The main peak value seems to be a poor tool for identifying 262 cracked capacitors. Fig. 5 does not suggest a clear trend be- 263 tween the main peak value and the likelihood of a crack. On 264 the other hand, a weak trend can be observed between the sec- 265 ondary peak value and the emergence of cracks. However, high 266 secondary peak values are not unambiguously an indication of 267 cracks. 


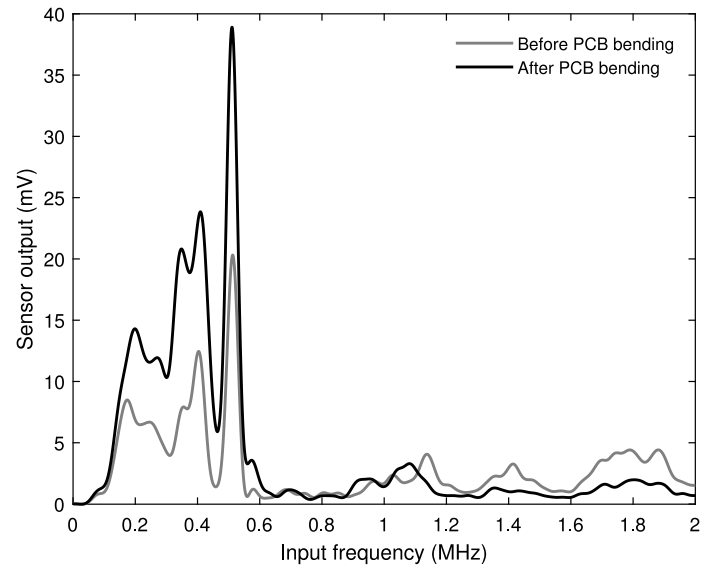

(a) Acoustic response of $\mathrm{C} 198$ before and after bending

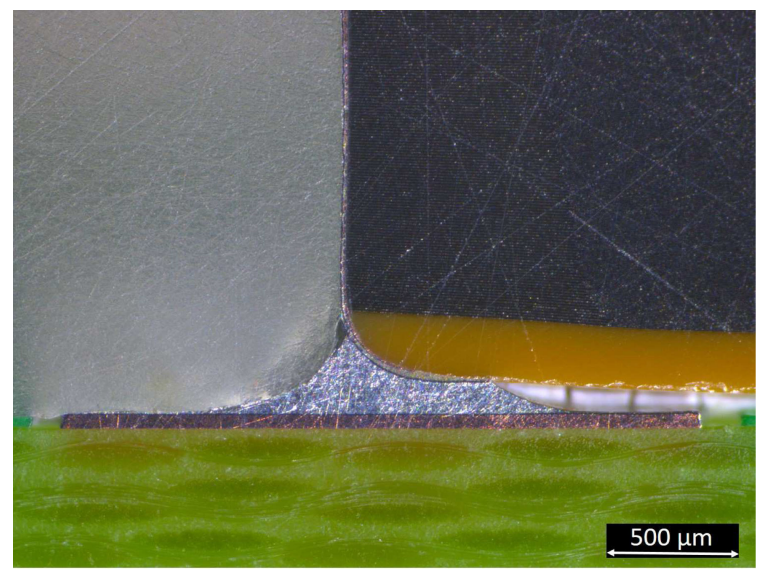

(b) Cross-section of C198 after bending. No cracks or delamination can be seen.

Fig. 2. Typical example of an MLCC with no observed damage after the bending of the PCB. Slight main peak amplitude increase is seen in (a) at around $500 \mathrm{kHz}$, but no new resonant peaks are introduced.

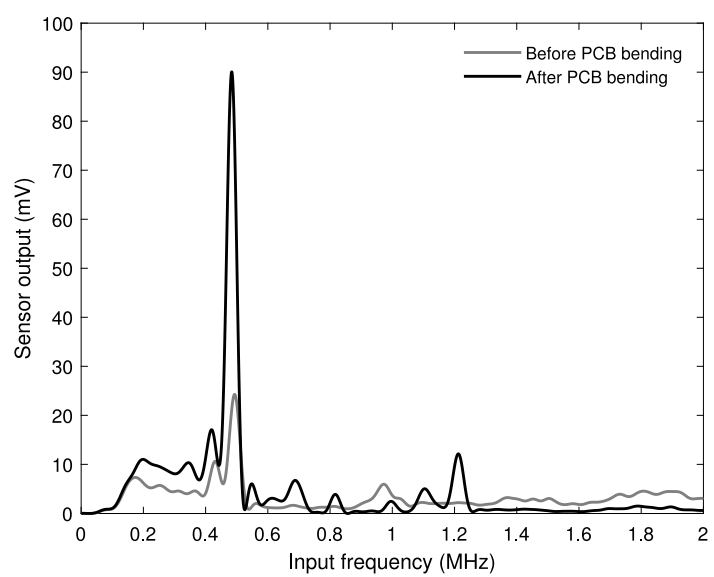

(a) Acoustic response of $\mathrm{C} 224$ before and after bending

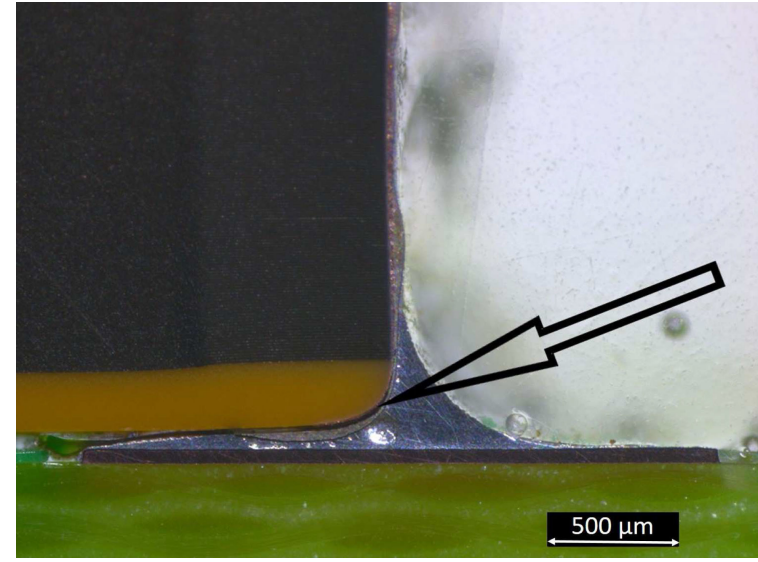

(b) Cross-section of $\mathrm{C} 224$ after bending, showing delamination under the right-hand-side termination.

Fig. 3. Typical example of a capacitor with delamination. The acoustic response in (a) shows significant increase in the main peak value, and several new peaks at different frequencies are introduced above $0.6 \mathrm{MHz}$.

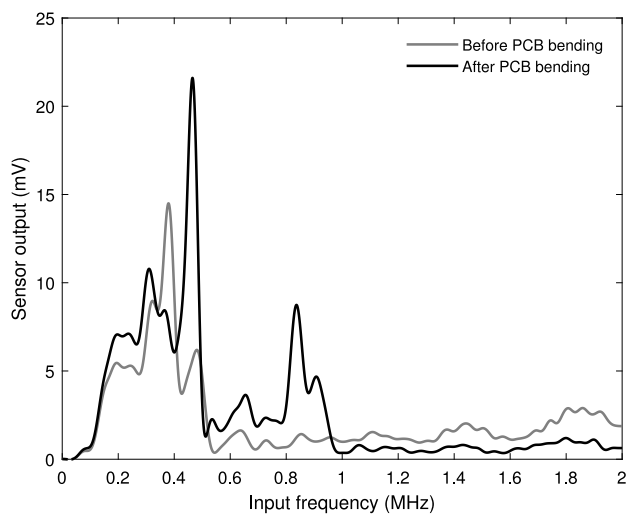

(a) Acoustic response of $\mathrm{C} 201$ before and after bending

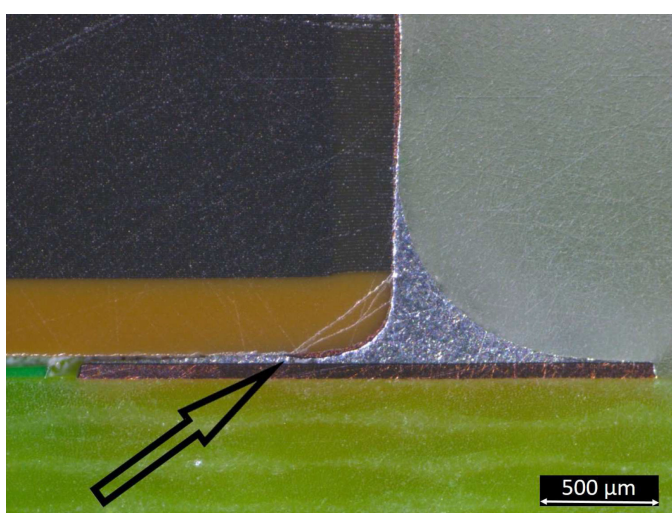

(b) Cross-section of C201 after bending, showing cracks in the passive area near the right-hand-side termination

Fig. 4. Typical example of an MLCC with multiple cracks near the termination. Main peak amplitude increase and new resonant peaks around $800 \mathrm{kHz}$ are seen in (a). 

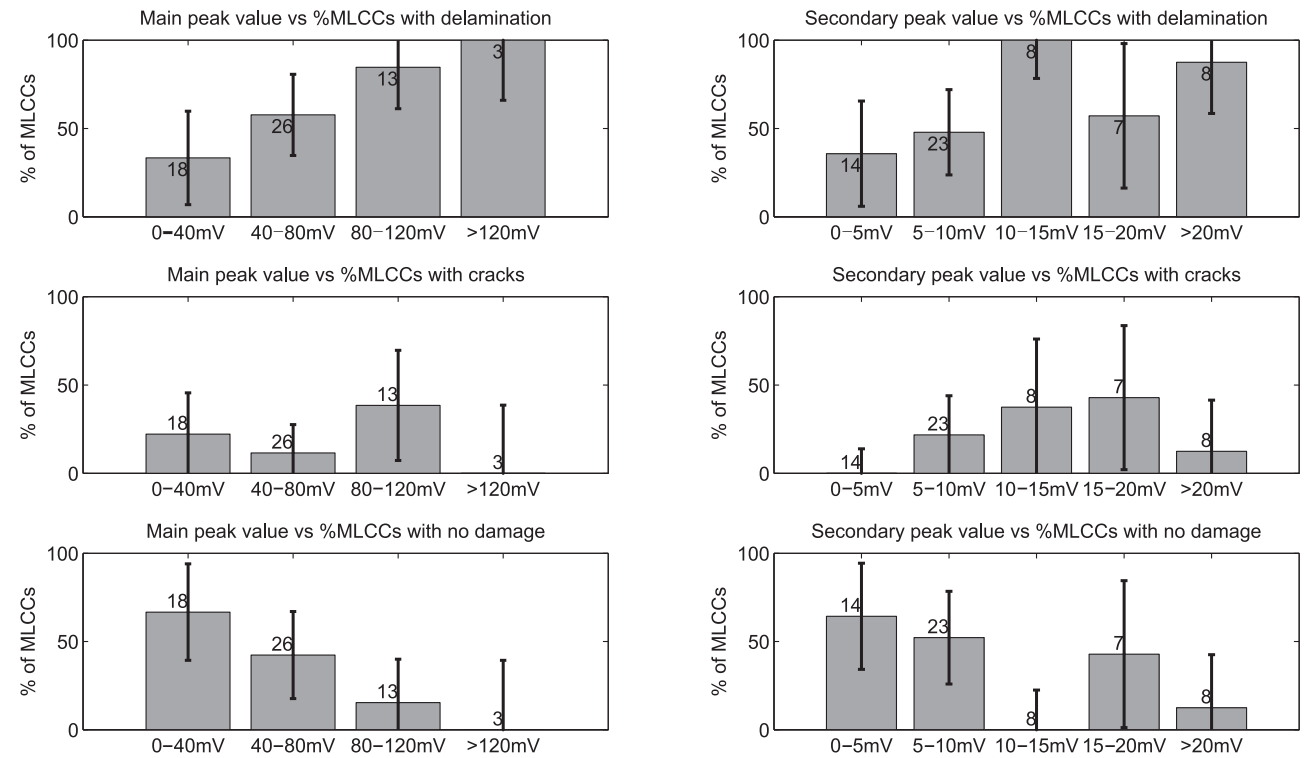

Fig. 5. Histograms describing the relationships amongst cracks, delamination, and acoustic emission peak values of 60 MLCCs. The bins are based on the peak values, and the numbers indicate the total amount of MLCCs in each bin. The error bars depict the combined uncertainty of measurement and sampling errors (see Appendix). Significant correlation between delamination and increased main peak value can be seen, whereas secondary peak values weakly correlate with the occurrence of cracks. Both peak values are generally lower for undamaged capacitors.

Undamaged MLCCs appear to exhibit lower values than damaged capacitors, both in terms of main and secondary peaks. This suggests that an increase in either of the peak values is a possible sign of damage.

\section{A. Detecting Damaged Capacitors Using SVM Classifiers}

In order to discriminate between damaged and undamaged MLCCs, an SVM classifier was trained on data extracted from 6 the acoustic measurements. Each MLCC was mapped onto 77 a two-dimensional feature space, with the features being the main and secondary peak values after the PCB bending. Each MLCC was labeled either damaged or undamaged, depending whether or not cracks and/or delamination were observed in the cross-sectioning. The SVM classifier was then trained by fitting a decision surface into the data set as shown in Fig. 6. A Gaussian kernel function was chosen for the SVM model, since it performed better than polynomial kernels. A linear SVM was 5 unable to separate the datapoints properly, whereas using poly6 nomial kernel functions caused the SVM model to overfit into 7 the data.

Because the data set consisted of only 60 MLCCs, dividing 9 it into separate training and validation sets would have had o significant negative effect on the performance of the classifier. 1 Instead, leave-one-out cross validation [31] was used to evaluate 2 the performance of the classifier: the SVM was trained on data 3 from all but one MLCC, and the performance of the classifier 4 was tested with the left-out MLCC. This procedure was repeated for all 60 MLCCs, simulating the performance of the SVM on data outside the training set.

In classifying undamaged capacitors, the SVM model performed equally well for both in-sample (training) and cross-validated data [see Fig. 7(a) and (b)]. In cross valida-

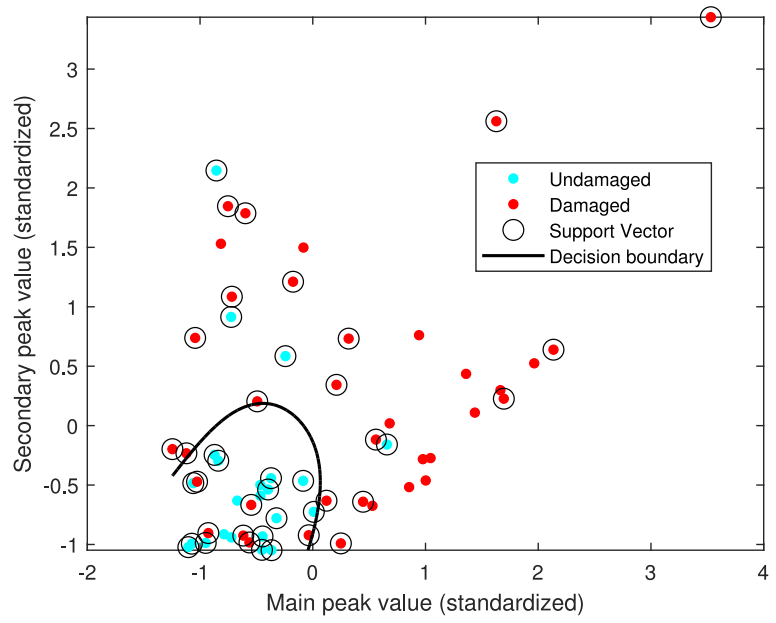

Fig. 6. SVM classifier fitted into observed MLCC features. The peak height data were obtained from the acoustic measurements after bending the PCB. In order to improve the classifier performance, the data were standardized to zero mean and unit variance. Capacitors which showed either delamination and/or cracks in the cross-sectioning are labeled as damaged.

tion, damaged MLCCs were missed by the classifier slightly 300 more often, while the SVM was still able to detect three out of 301 four damaged capacitors. The overall cross-validated accuracy 302 of the classifier was $78.3 \%$, whereas the recall (how many of 303 the damaged capacitors were found) was $75.7 \%$. 304

The operation of the classifier at different threshold values is 305 visualized by the receiver operating characteristic (ROC) [32] 306 curve in Fig. 8. With out-of-sample data, the SVM classifier 307 performs similarly as with data within the training set. The 308 area under ROC curve (AUROC) with out-of-sample data is 309 approximately 0.78 , indicating a fairly good classifier despite 310 the small amount of training data. A perfect classifier would 311 


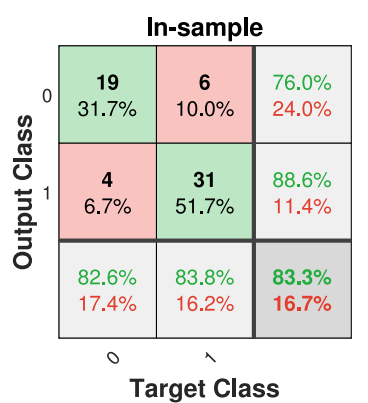

(a) Confusiuon matrix calculated with values within the training (insample) data

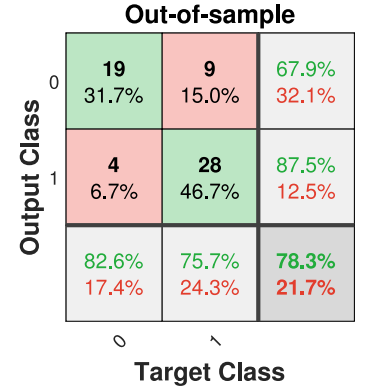

(b) Out-of-sample confusion matrix. The values are obtained by pooling the output from each leaveout-one cross-validated model
Fig. 7. SVM classifier confusion matrices for MLCCs in the training data set and combined cross-validation results.

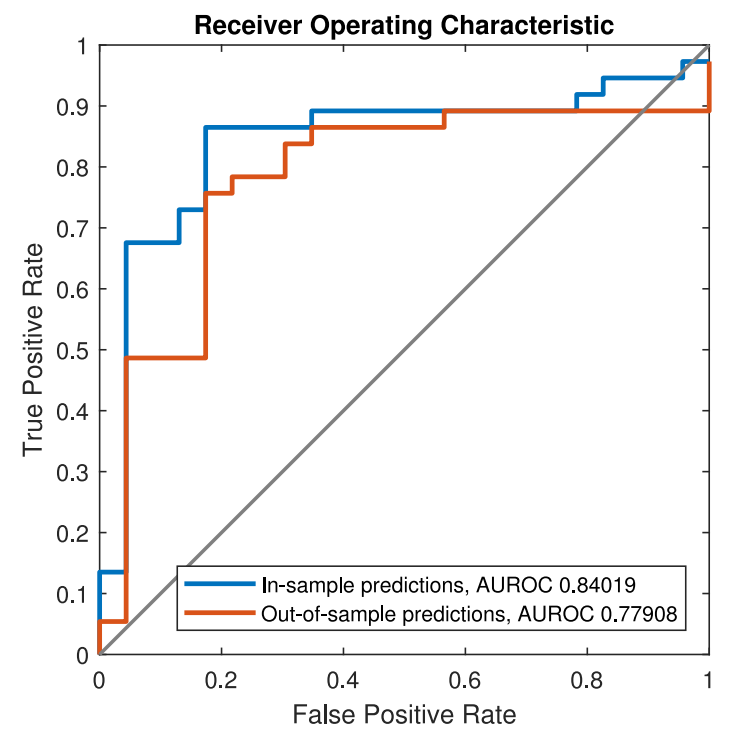

Fig. 8. ROC of the trained SVM classifier at different output threshold values. AUROC measures the overall performance of the classifier: a perfect classifier has an AUROC of 1.0, whereas pure guessing produces an AUROC of 0.5.

have an AUROC value of 1.0, whereas purely random guessing 313 would produce an AUROC of 0.5. The performance of the clas314 sifier could be improved by including more of the features in 315 the acoustic response, provided that the features actually carry 316 information about the condition of a capacitor.

317 The classification results presented here are for an SVM 318 trained to detect whether or not the capacitor had suffered 319 any kind of damage. Classifiers were also trained to detect 320 specifically either delamination or cracks. Delamination was de321 tected with only slightly lower performance than the combined 322 classifier. For cracks, however, the classifier output was neg323 ative for every single capacitor. Unlike in Fig. 6, the cracked 324 capacitors mapped onto the feature space formed no clear clus325 ter for the decision plane to separate from the rest of the data. 326 This suggests that while the main and secondary peak values are 327 enough to determine whether or not an MLCC is delaminated,

\section{DISCUSSION}

The data obtained in this study suggest that main peak value 330 may turn out to be a valuable metric in identifying delaminated 331 capacitors, especially when used together with secondary peak 332 value information. However, neither of these peak values seem 333 to explicitly indicate the presence of cracks.

In the analysis presented in this paper, only two peak values 335 are taken into account. As it is mentioned in Section III, the 336 secondary peak frequency is not fixed, and varies from capacitor 337 to capacitor. Considering how simple the extracted features are 338 and how small a portion of the frequency response is used, the 339 SVM classifier performs remarkably well. However, capacitors 340 containing only cracks were left undetected.

The determination of delamination and cracks presented in 342 Figs. 5 and 6 is not guaranteed to be error free. The data were 343 obtained by looking at cross section images of the capacitors, 344 and in such a process misidentification is a possibility. Training 345 the SVM model with misidentified capacitors would decrease its 346 accuracy. Nevertheless, the performance of the SVM classifier 347 surpassed the initial expectations of the authors based on visual 348 and statistical observations.

It is likely that by extracting proper features, such as more 350 resonant peaks and their frequency information, the classifier 351 performance would improve both for crack and delamination 352 detection. More flexible classifiers, such as neural networks or 353 random forests could also yield better results; this is a matter for 354 further study. Moreover, the acoustic approach could be used as 355 a complementary method to X-ray analysis, which can identify 356 cracks accurately, but not delaminations.

The cracks and delaminations in this study were relatively 358 small, the vast majority of them residing in the passive region 359 of the capacitors. Nevertheless, even a small fault in a capacitor 360 can grow over time, and water can get inside the component 361 with harmful results. Even though the dimensions of a damaged 362 region cannot be accurately defined by cross section images, 363 damage at the capacitor termination less than $500 \mu \mathrm{m}$ long can 364 cause changes in the acoustic signature.

Remarkably, the results were identical for flex and "normal" 366 termination capacitors. The authors expected that some differ- 367 ence would be seen between these capacitor types, but this 368 turned out not to be the case. From the production testing point 369 of view, this is a welcome result, as the test method would not 370 have to be redesigned for different termination types. 371

Even though the capacitors used in this study are physically 372 larger than those found in, e.g., consumer electronics, acous- 373 tic screening is applicable for case sizes of 1206 and below 374 [27]. It must also be noted that smaller capacitors are not as 375 prone to damage from bending [7]. Therefore, the most potential 376 applications of acoustic screening are in, e.g., power electron- 377 ics, where large case sizes and heavy circuit board assemblies 378 increase the risk of flex cracks and delamination. 379

The physical phenomena causing the changes in the acoustic 380 response has not been discussed yet. It seems likely that de- 381 lamination near the end terminations of an MLCC allows the 382 capacitor to vibrate more freely in a direction perpendicular to 383 the PCB surface. Because vibration in this direction creates the 384 
largest displacement for the point contact sensor, increase in main peak values is observed. Resonance peaks observed in the secondary peak region have lower amplitude, and thus are likely related to other resonance modes of the capacitor body. A possible explanation for this is that cracks in the ceramic body allow the MLCC to vibrate more freely along these modes, causing secondary peaks to appear.

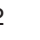

A set of MLCCs was investigated acoustically before and after inflicting damage. The changes in the acoustic characteristics were analyzed and compared against the damage observed in each capacitor. The acoustic data were also used to train an SVM classifier to detect damaged capacitors.

The main finding was that delamination of the capacitor causes quite consistent changes in the acoustic response of a capacitor. Cracks in the capacitor cause changes as well, but are not as easy to detect as those caused by delamination.

The SVM classifier showed promising performance. Nevertheless, further study on feature extraction and classifier algorithms is required to develop a reliable capacitor screening method based on acoustic emission.

APPENDIX

In order to calculate the error bars for Fig. 5, two major sources of measurement uncertainty were identified: 1) the misidentification of cracks and delaminations in the cross section images, and 2) the sampling error caused by the relatively low number of capacitors in many of the bins in Fig. 5.

Other sources of uncertainty, such as the uncertainty present in the measurement instruments, do exist in this case. It was estimated that their contribution to the total uncertainty of the measurement was insignificant when compared to the uncertainty of the identification of damage and the sampling error.

For the identification of cracks, it was estimated that the likeli8 hood of a false positive, i.e., an intact capacitor being incorrectly determined as having a crack, was $1 \%$. The likelihood of a false negative, i.e., a cracked capacitor being incorrectly determined as being intact, was estimated to be 5\%. Similarly, for the identification of delamination, the false positive rate was estimated as $5 \%$, and the true positive rate as $10 \%$.

Using these false identification rates, the maximum and minimum bounds of the error $a_{+}$and $a_{-}$were calculated. This yields an asymmetrical distribution of error, which is difficult to handle in the uncertainty estimation process. For this reason, these 8 bounds were converted into a single standard uncertainty value $u_{1}$ based on [33, Sec. 4.3.8] as

$$
u_{1}^{2}=\frac{a_{+}-a_{-}}{12} .
$$

To estimate the effect of sampling error, upper and lower bounds were taken from the chart in [34, p. 494]. In this case, the distribution is heavily skewed, especially in the bins where the number of capacitors is low. The obtained up4 per and lower bounds were again used to obtain a single standard uncertainty value

$$
u_{2}^{2}=\frac{a_{+}-a_{-}}{12} .
$$

The two standard uncertainty values were then merged into a 436 combined uncertainty

$$
u_{c}=\sqrt{u_{1}^{2}+u_{2}^{2}}
$$

which was then multiplied with a coverage factor $k=1.960$ to 438 expand the uncertainty to a confidence level of $95 \%$. The bar 439 height in Fig. 5 is the observed percentage of capacitors $p$ in 440 each bin, and the error bars cover the range $p \pm k u_{c}$. The error 441 bars are cut at $100 \%$ and $0 \%$ because values outside these values 442 are not meaningful.

The authors would like to note that this analysis is heavily 444 simplified. The resulting uncertainty estimates are taken to be 445 good enough for an understanding of how well the results can 446 be generalized to another, similar capacitor population.

\section{ACKNOWLEDGMENT}

The authors would like to thank J.-Y. Loisy and S. T. Yildiz at 449 ABB Corporate Research for cross-section sample preparation 450 and imaging by optical microscopy.

\section{REFERENCES}

[1] B.-H. Ko, S.-G. Jeong, Y.-G. Ahn, K.-S. Park, N.-C. Park, and Y.-P. 453 Park, "Analysis of the correlation between acoustic noise and vibration 454 generated by a multi-layer ceramic capacitor," Microsyst. Technol., vol. 20, 455 nos. 8/9, pp. 1671-1677, 2014.

[2] C.-W. Huang, B.-T. Chen, K.-Y. Chen, C.-H. Hsueh, W.-C. Wei, and 457 C.-T. Lee, "Finite element analysis and design of thermal-mechanical 458 stresses in multilayer ceramic capacitors," Int. J. Appl. Ceramic Tech- 459 nol., vol. 12, no. 2, pp. 451-460, Mar. 2015. [Online]. Available: 460 http://search.proquest.com/docview/1660318462?accountid=136582. 461

[3] T. Adams, "Preventing MLCC failures," Circuits Assembly, pp. 17-19, 462 Aug. 20, 2009. [Online]. Available: https://www.circuitsassembly.com/ 463 ca/editorial/menu-features/296-current-articles/17452-preventing-mlcc- 464 failures.html

[4] J. A. Ahmar and S. Wiese, "A finite element modelling and fracture 466 mechanical approach of multilayer ceramic capacitors," in Proc. 16th Int. 467 Conf. Thermal, Mech. Multi-Physics Simul. Experiments Microelectronics 468 Microsystems, Apr. 2015, pp. 1-5.

[5] T. Adams, "High acoustic frequency imaging," Ceramic Ind., vol. 164, 470 no. 2, pp. 14-16, 2014. [Online]. Available: http://search.ebscohost. 471 com/login. aspx $?$ direct $=$ true $\& d b=$ bth $\& A N=94147595 \&$ site $=$ ehost-live 472

[6] S. R. Kahn and R. W. Checkaneck, "Acoustic emission testing of multi- 473 layer ceramic capacitors," IEEE Trans. Compon., Hybrids, Manuf. Tech- 474 nol., vol. CHMT-6, no. 4, pp. 517-526, Dec. 1983.

[7] M. Keimasi, M. H. Azarian, and M. G. Pecht, "Flex cracking of multilayer 476 ceramic capacitors assembled with pb-free and tinlead solders," IEEE 477 Trans. Dev. Mater. Rel., vol. 8, no. 1, pp. 182-192, Mar. 2008.

[8] J. M. J. den Toonder, C. W. Rademaker, and C.-L. Hu, "Residual stresses 479 in multilayer ceramic capacitors: Measurement and computation," J. Elect. 480 Packag., vol. 125, no. 4, pp. 506-511, Dec. 2003.

[9] G. Vogel, "Avoiding flex cracks in ceramic capacitors: Analytical tool for 482 a reliable failure analysis and guideline for positioning cercaps on PCBs," 483 Microelectron. Rel., vol. 55, no. 9, pp. 2159-2164, 2015.

[10] C. Andersson, J. Ingman, E. Varescon, and M. Kiviniemi, "Detec- 485 tion of cracks in multilayer ceramic capacitors by X-ray imaging," 486 Microelectron. Rel., vol. 64, pp. 352-356, 2016. [Online]. Available: 487 http://www.sciencedirect.com/science/article/pii/S0026271416302542 488

[11] L. Bechou, S. Mejdi, Y. Ousten, and Y. Danto, "Non-destructive detection 489 and localization of defects in multilayer ceramic chip capacitors using 490 electromechanical resonances," Quality Rel. Eng. Int., vol. 12, pp. 43-53, 491 1996. 37 
[12] V. Krieger, W. Wondrak, A. Dehbi, W. Bartel, Y. Ousten, and B. Levrier, "Defect detection in multilayer ceramic capacitors," Microelectron. Rel., vol. 46, pp. 1926-1931, 2006.

13] Y. C. Chan, F. Yeung, G. Jin, N. Bao, and P. S. Chung, "Nondestructive detection of defects in miniaturized multilayer ceramic capacitors using digital speckle correlation techniques," IEEE Trans. Compon., Packag., Manuf. Technol. A, vol. 18, no. 3, pp. 677-684, Oct. 1995. [Online]. Available: http://search.proquest.com/docview/27455226?accountid $=136582$

[14] D. L. Commare, "Nondestructive evaluation of MLCCs," Ceramic Ind. vol. 140, no. 6, pp. 38-41, 1993.

[15] G. F. Kieran, "A comparison of screening techniques for ceramic capacitors," in Proc. Symp. NASA Conf. Capacitor Technol., Appl. Rel., 1981, pp. 111-117.

[16] J. D. Prymak, "Piezoelectric effects ceramic chip capacitors (singing capacitors)," Arrow Asian Times article, KEMET Electronics Corporation, Aug. 2006. [Online]. Available: http://www.kemet.com/Lists/ TechnicalArticles/Attachments/88/2006\%2007\%20ArrowAsiaTimes\% 20-\%20MLC\%20Noise.pdf

[17] J. Chen and Z. Feng, "X7r dielectric multilayer ceramic capacitors show good micro-actuating properties with little hysteresis," Electron. Lett., vol. 50, no. 7, pp. 538-540, Mar. 2014.

[18] S. Levikari, T. J. Kärkkäinen, C. Andersson, J. Tamminen, and P. Silventoinen, "Acoustic phenomena in damaged ceramic capacitors," IEEE Trans. Ind. Electron., vol. 65, no. 1, pp. 570-577, Jan. 2018.

19] W. L. Johnson, S. A. Kim, G. S. White, and J. Herzberger, "Nonlinear resonant acoustic detection of cracks in multilayer ceramic capacitors," in Proc. IEEE Int. Ultrason. Symp., Sep. 2014, pp. 244-247.

[20] W. L. Johnson, S. A. Kim, G. S. White, J. Herzberger, K. L. Peterson, and P. R. Heyliger, "Time-domain analysis of resonant acoustic nonlinearity arising from cracks in multilayer ceramic capacitors," in Proc. AIP Conf. Proc., 2016, vol. 1706.

[21] W. I. Johnson, J. L. Herzberger, S. A. Kim, K. L. Peterson, P. R. Heyliger, and G. S. White, "Resonant acoustic frequency shifts associated with cracks in multilayer ceramic capacitors," IEEE Trans. Device Mater. Rel. vol. 17, no. 2, pp. 316-323, Jun. 2017.

[22] T. J. Kärkkäinen, J. P. Talvitie, O. Ikonen, M. Kuisma, P. Silventoinen, and E. Mengotti, "Sounds from semiconductors - acoustic emission experiment with a power module," in Proc. 16th Eur. Conf. Power Electron. Appl., Aug. 2014, pp. 1-6.

[23] T. J. Kärkkäinen et al., "Acoustic emission in power semiconductor modules - first observations," IEEE Trans. Power Electron., vol. 29, no. 11, pp. 6081-6086, Nov. 2014.

[24] T. J. Kärkkäinen, J. P. Talvitie, M. Kuisma, P. Silventoinen, and E. Mengotti, "Acoustic emission caused by the failure of a power transistor," in Proc. IEEE Appl. Power Electron. Conf. Expo., Mar. 2015, pp. 24812484.

[25] C. Cortes and V. Vapnik, "Support-vector networks," Mach. Learn. vol. 20, no. 3, pp. 273-297, Sep. 1995. [Online]. Available: https://doi.org/10.1007/BF00994018

[26] S. Levikari, T. J. Kärkkäinen, P. Silventoinen, C. Andersson, and J. Tamminen, "Acoustic detection of cracks and delamination in multilayer ceramic capacitors," in Proc. IEEE 11th Int. Symp. Diagnostics Electr. Mach. Power Electron. Drives, Aug. 2017, pp. 622-627.

[27] S. Levikari, "Detection of cracks: Acoustic experiments on multilayer ceramic capacitors," M.S. thesis, Lappeenranta Univ. Technol., Lappeenranta, Finland, 2018

[28] S. Levikari, T. J. Kärkkäinen, C. Andersson, and J. Tamminen, "MLCC acoustic data set," Lappeenranta Univ. Technol., Lappeenranta, Finland, 2018. [Online]. Available:http://urn.fi/urn:nbn:fi:csckata20180302153714692880.

[29] S. Siuly, Y. Li, and Y. Zhang, EEG Signal Analysis and Classification New York, NY, USA: Springer, 2016

[30] M. Li, X. Liu, and X. Liu, "Infrasound signal classification based on spectral entropy and support vector machine," Appl. Acoust., vol. 113, pp. 116-120, 2016. [Online]. Available: http://www.sciencedirect.com/ science/article/pii/S0003682X16301761

[31] G. Dougherty, Pattern Recognition and Classification: An Introduction. New York, NY, USA: Springer, 2013, ch. 9.

[32] T. Fawcett, "An introduction to ROC analysis," Pattern Recognit. Letters, vol. 27 , no. 8, pp. 861-874, 2006.

[33] JCGM, 100:2008 Evaluation of Measurement Data-Guide to the Expression of Uncertainty in Measurement, corrected version 2010 ed. JCGM, 2010.

34] L. Råde and B. Westergren, Mathematics Handbook for Science and Engineering. Lund, Sweden: Studentlitteratur, 1988, 2004.

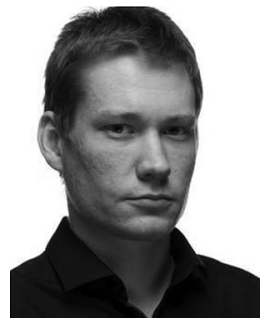

Saku Levikari (M'18) was born in 1991 in Fin- 568 land. He received the B.Sc. and M.Sc. degrees 569 from Lappeenranta University of Technology (LUT), 570 Lappeenranta, Finland, in May and June of 2018, 571 respectively.

$\mathrm{He}$ is currently a Doctoral Student with LUT 573 School of Energy Systems, Laboratory of Applied 574 Electronics. His main research topics include the field 575 of data analytics and machine learning, with main 576 focus on wearable electronics and the reliability of 577 power electronics.

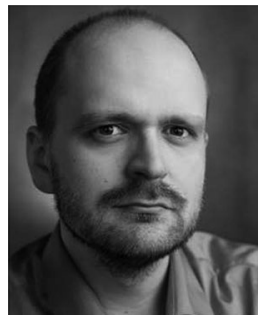

Tommi J. Kärkkäinen (M'12) was born in 1987580 in Finland. He received the B.Sc., M.Sc., and D.Sc. 581 degrees from Lappeenranta University of Technology 582 (LUT), Lappeenranta, Finland, in 2010, 2011, and 583 2015, respectively.

$\mathrm{He}$ is currently a Postdoctoral Researcher with 585 LUT School of Energy Systems. His main research 586 topic is the reliability of power electronic devices 587 and systems, and the utilization of machine learning 588 algorithms to improve the reliability and productivity 589 of various systems.

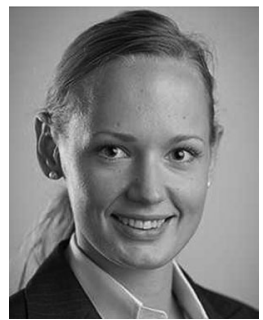

Caroline Andersson (M'10) was born 1983 in Swe- 592 den. She received the M.Sc. degree from Lund 593 University, Lund, Sweden, and the Ph.D. degree 594 from Swiss Federal Institute of Technology, Zurich, 595 Switzerland.

She is currently a Senior Scientist with ABB Cor- 597 porate Research Center, Baden, Switzerland, on the 598 topic of reliability of power electronics. The main 599 focus of her research area is accelerated life testing, 600 physics of failure, and reliability of components and 601 systems. 602

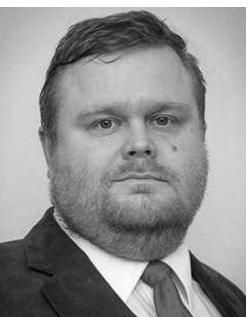

Juha Tamminen was born in 1980 in Finland. He 604 received the B.Eng. degree in electronics in 2006605 from Espoo-Vantaa University of Applied Sciences, 606 Vantaa, Finland.

$\mathrm{He}$ is currently a Design Manager with ABB 608 Drives, Helsinki, Finland, in the field of production 609 testing. His work concentrates on improving produc- 610 tion testing concepts in pursue of better quality and 611 productivity.

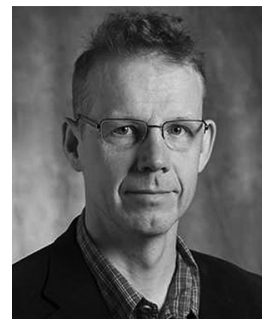

Pertti Silventoinen (M'09) was born in Simpele, 614 Finland, in 1965. He received the D.Sc. degree from 615 Lappeenranta University of Technology, Lappeen- 616 ranta, Finland, in 2001.

He became a Professor of applied electronics in 618 2004. His current research interests include power 619 electronics systems in various applications. $\quad 620$ 